\title{
Growth and Biochemical performance of Cassava-Manihot esculenta Crantz to Crude oil Polluted soil amended with Centrosema pubescens Benth and NPK
}

\author{
*11EREMRENA, P.O.; AKONYE, L. A. \\ ${ }^{a}$ Department of Plant Science and Biotechnology, Faculty of Biological Science, College of Natural and Applied Sciences, University of \\ Port Harcourt, P.M.B.5323, Choba Port Harcourt, Rivers State, Nigeria.
}

Key words: Growth, Manihot esculenta, crude oil, pollution, Amelioration

\begin{abstract}
Growth and biochemical performance of Manihot esculenta Crantz (TMS 0505) amended with Centrosema pubescens and NPK fertilizer were carried out. Contamination levels of $2 \%, 4 \%$, and $6 \%$ were used while amelioration treatments $C$. pubescens and NPK 15:15:15 were carried out by adding organic supplement (decomposed Centrosema pubescens) and NPK at the rate of $0.25 \mathrm{Kg}$ per $5 \mathrm{Kg}$ of soil to the various levels of crude oil contaminated soil, alongside a control treatment. Results showed that addition of Centrosema pubescens and NPK to the crude oil polluted soil improved the growth and biochemical performance of cassava. For the qualitative observations tested, the amelioration treatment recorded significant $(\mathrm{P}=0.05)$ increase in plant height, petiole length, leaf number, leaf area, fresh weight, dry weight and moisture content, than those of the contamination treatment. Soil physico-chemical analysis such as Total Organic Carbon (TOC), Total Organic Matter (TOM), pH, available phosphorus, Total Nitrogen and heavy metals analysis were determined in the soil before and after pollution and after amelioration. There were significant $(\mathrm{P}=0.05)$ increases in plant height, leaf number, leaf area, dry weight, fresh weight and total Chlorophyll contents in soils amended with both C. pubescens and NPK fertilizer than those containing single amendment (C. pubescens or NPK fertilizer). Therefore, this study suggests that Centrosema pubescens and NPK in combination proved effective in remediation of the growth components of Manihot esculenta in crude oil contaminated soil. CJASEM
\end{abstract}

\section{DOI: http://dx.doi.org/10.4314/jasem.v17i2.4}

Human technological and scientific advances have caused environmental changes that are impossible to evaluate and fully comprehend. Our ability to change the environment has increased faster than the ability to predict the effect of that change. Pollution of the environment is one of the major effects of human technological advancement. Pollution results when a change in the environment harmfully affects the quality of human life including effects on animals, microorganisms and plants (Ogboghodo, et al.,2005). Crude oil pollution can be defined as the introduction of crude oil or its derivatives with its associated gases into the environment (air, water and land) in quantities that are poisonous or capable of causing immediate physical, chemical and biological damage to the affected ecosystem (Tanee \& Anyanwu, 2007). One of the environmental challenges posed by this oil pollution is the alteration in the physical and chemical nature of the soil which subsequently affects the growth of plants (Tanee and Akonye, 2009). Petroleum hydrocarbon contamination may affect plant by retarding seed germination and reducing plant height, stem density, photosynthetic rate and biomass or resulting in complete mortality ( Pezeshki, et al.,2000).

Petroleum (crude oil) pollution has been found to affect the cultivation and production of economic crops including cassava especially in the Niger Delta. There is therefore, need to remediate such polluted site to improve plant performances.

The study attempts to investigate the growth performance of cassava (var. TMS 0505) in crude oil contaminated soil amended with leaves of Centrosema pubescens and NPK. The choice of cassava for this study is necessitated by the fact that it is the most common crop cultivated in the Niger Delta where crude oil pollution is inevitable. It is expected that result obtained from this study will widen our knowledge on the effect of oil pollution on the growth of plants and how Centrosema pubescens and NPK fertilizer can be use to improve such conditions for better performance of crops.

\section{MATERIALS AND METHODS}

The top loamy soil used for the study were collected from the University of Port Harcourt Botanic Garden. The crude oil was obtained from Nigerian National Petroleum Corporation, Eleme, Rivers State. Cassava (Manihot esculenta Crantz) TMS 0505 variety cuttings and NPK 15:15:15 were obtained from Agricultural Development Programme (ADP) in Rivers State. Leaves of Centrosema pubescens were obtained from farms at Alakahia community opposite University of Port Harcourt Teaching Hospital (UPTH) and Abuja campus. physico-chemical properties of the experimental soils (loamy soil, and contaminated soil) were analyzed using standared procedure (Allison, 1965; A.O.A.C., 1975; Udo and Ogunwale, 1986).

A 3x4 factorial arrangement fitted into completely randomized design were used, each treatment were replicated three times. Top loamy soil weighing $5 \mathrm{~kg}$ for each bucket were used. The soil was mixed thoroughly with different levels of crude oil thus, $0 \%$, $2.0 \%, 4 \%$ and $6 \%$ and placed in a plastic buckets 
based on each treatment. A total of 72 plastic buckets were used for the experiment. The buckets were perforated at the bases and sides to allow for aeration and drainage. These were allowed to stand for one week for the oil to acclimatized to the soil before remediation.

Leaves of Centrosema pubescens and NPK 15:15:15 were used to ameliorate the crude oil contaminated soil. The reasons for the choice of these two materials are that they are cheap, easily available and have high nitrogen content which is always a limiting factor in a crude oil polluted soil. Each ameliorating material was weighed $0.25 \mathrm{~kg}$ for each $5 \mathrm{~kg}$ soil per bucket. The remediation/treatments were in this order,

Soil with $0 \%, 2 \%, 4 \%$ and $6 \%$ crude oil contamination respectively, Soil with $0 \%, 2 \%, 4 \%$, $6 \%$ crude oil contamination and NPK, Soil with $0 \%$, $2 \%, 4 \%$ and $6 \%$ of crude oil and C. pubescens, Soil with $0 \%, 2 \%, 4 \%$ and $6 \%$ of crude oil mixed with $C$. pubescens and fertilizer

After treatment was carried out, a period of three weeks was allowed for the NPK and leaves of Centrosema pubescens to decompose in the soil before planting. Three cassava cuttings, ranging from $4-5 \mathrm{~cm}$ were planted thereafter

The following growth and biochemical parameters were analysed: plant height, petiole length, leaf number, fresh weight, dry weight, total organic carbon (TOC), total organic matter (TOM), $\mathrm{pH}$, available phosphorus, Total Nitrogen and heavy metals.

The shoot length (plant height) was measured with a metre tape in centimetres from the soil surface to the plant apex. The plant were uprooted from each bucket and weighed immediately on a weighing balance, model PN 163 to avoid moisture loss. This was done to obtained the fresh weights. To get the dry weights, the plants were taken to the laboratory, oven-dried at $80^{\circ} \mathrm{C}$ for 24 hours to get rid of all moisture and ensure a constant weight. It was then weighed on a PN 163 model weighing balance. Leaf chlorophyll content was extracted from $1.0 \mathrm{~g}$ of leaf sample. The sample was homogenized by adding small amount of $85 \%$ acetone. $25 \mathrm{ml}$ aliquot of extract was added to $50 \mathrm{ml}$ diethyl ether in a separating funnel. The optical density at $660 \mathrm{~nm}$ and $643 \mathrm{~nm}$ in $1 \mathrm{~cm}$ cell was measured using ether as a reference. Leaf carbohydrate content was analysed by extracting $1.0 \mathrm{~g}$ of dry leaf sample and digested with Perchloric acid and the sugar was determined colorimetrically by the Anthrone method. The nitrogen content was determined by the Kjedahl method (Stewart, et $a l ., 1974)$ in which $1.0 \mathrm{~g}$ of leaf sample was heated on an electro thermal hot plate, until digest turned to sky-blue, then diluted with $100 \mathrm{mls}$ of diluted water. $30 \mathrm{mls}$ of $40 \% \mathrm{NaOH}$ added and the sample was heated to release ammonia. The distillate was titrated with $0.01 \mathrm{M}$ hydrochloric acid.

All data collected were subjected to statistical analysis such as Analysis of variance (ANOVA) and standard error means. New Duncan Multiple range test (NDMRT) was employed to separate means.

RESULTS AND DISCUSSION Result showed that addition of amendment materials to the crude oil polluted soil significantly $(\mathrm{P}=0.05)$ increased the plant height of cassava (fig.1). Highest significant plant height was recorded in the compost and NPK combined treatment. There were significant $(\mathrm{P}=0.05)$ reductions in petiole length and leaf number with increasing concentration of crude oil (fig. 2 and 3). In fig. 4 the fresh weight of cassava were found to improve with time when compared with the control. Compost and NPK 15:15:15 was found to be most effective in improving fresh weight yield than the other treatment options while the control produced least. Similar results were recorded for the dry weight (fig. 5)

Addition of ameliorating materials to crude oil polluted soil also improved the biochemical properties of cassava. Tables 1,2, 3 and 4 showed a significant $(\mathrm{P}=0.05)$ improvement in the leaf chlorophyll, leaf carbohydrate and leaf nitrogen in the different remediation treatment as compared to the control (no remediation). NPK 15:15:15 + Compost treatment recorded the highest $(\mathrm{P}=0.05)$ leaf chlorophyll content followed by compost. In leaf nitrogen content compost recorded the highest followed by NPK + Compost with no significant difference between them.

The drastic reductions in shoot length and root length as well as the entire stunted growth of plant in crude oil polluted soil may be due to high degree of toxicity causing damage to the root and shoot tissues thereby making the absorption of nutrients impossible (Udo and Oputa, 1984; Renault et al., 2000). Crude oil pollution also affected the leaf area of the crop, this may be as a result of interference of the oil constituent with photosynthesis and transpiration probably by clogging the stomata (Al-Azab et al., 2005). The concentration of crude oil pollution influenced the yield parameters of the crop at low and high pollution levels, respectively, compared to the controls. (Offor and Akonye, 2006). It may be suggested that the reduction in crop yield could have been due to competition for the limited nutrients between microbial population and the crop as a result of high carbon content in the soil relative to nitrogen (Inoni, 2006; Fernet, 2008), as depicted from the 
physico-chemical properties of the polluted soil in this study. In oil polluted soils, nitrogen and phosphorus quickly become limiting to biodegradation of oil and plant growth, resulting in a delay in the rate of soil recovery and a decrease in crop yield (Vwioke and Fashemi, 2005). It has been reported that addition of Nitrogen and phosphorus enhances biodegradation of polluted soil presumably by removing the Nitrogen and Phosphorus limitation resulting from low natural level (Lee et al.,2000; Odokuma and Ibor, 2002).

Table 1: Physico Chemical Properties Of Experimental Soil

\begin{tabular}{|c|c|c|c|c|}
\hline & \multicolumn{4}{|c|}{ CONCENTRATION (\%) } \\
\hline PARAMETERS & 0 & 2 & 4 & 6 \\
\hline $\mathrm{pH}$ & $5.7 \pm 0.10$ & $5.6 \pm 0.20$ & $5.3 \pm 0.13$ & $5.0 \pm 0.12$ \\
\hline Total N(\%) & $0.42 \pm 0.02$ & $0.25 \pm 0.04$ & $0.17 \pm 0.09$ & $0.05 \pm 0.01$ \\
\hline Available P (mg/100g) & $10.5 \pm 0.52$ & $5.27 \pm 0.23$ & $3.92 \pm 0.21$ & $3.07 \pm 0.19$ \\
\hline $\operatorname{Mn}(\mathrm{mg} / 100 \mathrm{~g})$ & $1.99 \pm 0.17$ & $2.12 \pm 0.21$ & $2.97 \pm 0.23$ & $3.16 \pm 0.30$ \\
\hline $\mathrm{Zn}(\mathrm{mg} / 100 \mathrm{~g})$ & $1.37 \pm 0.32$ & $1.26 \pm 0.29$ & $1.07 \pm 0.20$ & $1.02 \pm 0.18$ \\
\hline $\mathrm{Cu}(\mathrm{mg} / 100 \mathrm{~g})$ & $2.16 \pm 0.32$ & $1.37 \pm 0.22$ & $1.24 \pm 0.24$ & $1.16 \pm 0.19$ \\
\hline $\mathrm{Pb}(\mathrm{mg} / 100 \mathrm{~g})$ & $2.70 \pm 0.30$ & $1.92 \pm 0.21$ & $1.76 \pm 0.20$ & $1.52 \pm 0.18$ \\
\hline $\mathrm{Fe}(\mathrm{mg} / 100 \mathrm{~g})$ & $2.26 \pm 0.21$ & $2.94 \pm 0.27$ & $3.04 \pm 0.30$ & $3.46 \pm 0.40$ \\
\hline $\mathrm{Cd}(\mathrm{mg} / 100 \mathrm{~g})$ & $1.37 \pm 0.12$ & $1.62 \pm 0.18$ & $1.75 \pm 0.19$ & $1.79 \pm 0.20$ \\
\hline $\mathrm{Cr}(\mathrm{mg} / 100 \mathrm{~g})$ & $1.16 \pm 0.13$ & $1.27 \pm 0.16$ & $1.56 \pm 0.21$ & $1.62 \pm 0.25$ \\
\hline $\mathrm{K}(\mathrm{mg} / 100 \mathrm{~g})$ & $10.64 \pm 0.52$ & $7.36 \pm 0.45$ & $4.04 \pm 0.30$ & $3.21 \pm 0.29$ \\
\hline $\mathrm{Na}(\mathrm{mg} / 100 \mathrm{~g})$ & $41.05 \pm 0.67$ & $42.31 \pm 0.56$ & $42.87 \pm 0.46$ & $45.72 \pm 0.69$ \\
\hline $\mathrm{Mg}(\mathrm{mg} / 100 \mathrm{~g})$ & $5.20 \pm 0.42$ & $5.77 \pm 0.45$ & $5.98 \pm 0.52$ & $6.13 \pm 0.30$ \\
\hline $\mathrm{Ca}(\mathrm{mg} / 100 \mathrm{~g})$ & $5.59 \pm 0.24$ & $5.62 \pm 0.30$ & $5.97 \pm 0.28$ & $6.02 \pm 0.35$ \\
\hline Total organic C $(\%)$ & $1.20 \pm 0.18$ & $3.52 \pm 0.26$ & $4.06 \pm 0.35$ & $5.29 \pm 0.28$ \\
\hline Total organic matter $(\%)$ & $2.74 \pm 0.10$ & $2.17 \pm 0.20$ & $1.06 \pm 0.19$ & $0.45 \pm 0.05$ \\
\hline Sand $(\%)$ & $78.02 \pm 0.82$ & $76.21 \pm 0.76$ & $74.30 \pm 0.57$ & $73.20 \pm 0.59$ \\
\hline Silt $(\%)$ & $16.50 \pm 0.34$ & $12.20 \pm 0.26$ & $14.10 \pm 0.30$ & $14.24 \pm 0.26$ \\
\hline Clay (\%) & $5.20 \pm 0.23$ & $10.27 \pm 0.45$ & $11.02 \pm 0.56$ & $12.36 \pm 0.58$ \\
\hline Moisture content (\%) & $62.42 \pm 0.82$ & $57.61 \pm 0.50$ & $48.06 \pm 0.48$ & $42.16 \pm 0.38$ \\
\hline
\end{tabular}

Mean \pm Standard Error

Table 2:Physico Chemical Properties Of Polluted Soil After

\begin{tabular}{|ccccc|}
\hline & & \multicolumn{2}{c|}{ CONCENTRATION $(\%)$} & 6 \\
PARAMETERS & 0 & 2 & 4 & $5.0 \pm 0.18$ \\
$\mathrm{pH}$ & $5.6 \pm 0.12$ & $5.5 \pm 0.24$ & $5.4 \pm 0.18$ & $0.02 \pm 0.04$ \\
Total N(\%) & $0.32 \pm 0.05$ & $0.10 \pm 0.06$ & $0.07 \pm 0.08$ & $0.74 \pm 0.24$ \\
Available P $(\mathrm{mg} / 100 \mathrm{~g})$ & $6.70 \pm 0.58$ & $1.16 \pm 0.25$ & $1.02 \pm 0.25$ & $1.13 \pm 0.45$ \\
$\mathrm{Mn}(\mathrm{mg} / 100 \mathrm{~g})$ & $1.04 \pm 0.20$ & $1.05 \pm 0.25$ & $1.08 \pm 0.25$ & $0.32 \pm 0.22$ \\
$\mathrm{Zn}(\mathrm{mg} / 100 \mathrm{~g})$ & $0.87 \pm 0.32$ & $0.46 \pm 0.32$ & $0.38 \pm 0.26$ & $0.47 \pm 0.18$ \\
$\mathrm{Cu}(\mathrm{mg} / 100 \mathrm{~g})$ & $1.26 \pm 0.35$ & $0.61 \pm 0.24$ & $0.52 \pm 0.26$ & $0.28 \pm 0.20$ \\
$\mathrm{~Pb}(\mathrm{mg} / 100 \mathrm{~g})$ & $0.72 \pm 0.34$ & $0.46 \pm 0.26$ & $0.33 \pm 0.24$ & $1.76 \pm 0.55$ \\
$\mathrm{Fe}(\mathrm{mg} / 100 \mathrm{~g})$ & $1.60 \pm 0.25$ & $1.67 \pm 0.32$ & $1.72 \pm 0.35$ & $1.04 \pm 0.65$ \\
$\mathrm{Cd}(\mathrm{mg} / 100 \mathrm{~g})$ & $0.77 \pm 0.18$ & $0.85 \pm 0.30$ & $0.92 \pm 0.24$ & $1.12 \pm 0.30$ \\
$\mathrm{Cr}(\mathrm{mg} / 100 \mathrm{~g})$ & $0.89 \pm 0.16$ & $0.92 \pm 0.19$ & $1.05 \pm 0.38$ & $1.52 \pm 0.20$ \\
$\mathrm{~K}(\mathrm{mg} / 100 \mathrm{~g})$ & $7.46 \pm 0.53$ & $1.97 \pm 0.54$ & $1.73 \pm 0.35$ & $33.26 \pm 0.76$ \\
$\mathrm{Na}(\mathrm{mg} / 100 \mathrm{~g})$ & $27.41 \pm 0.70$ & $29.06 \pm 0.45$ & $32.17 \pm 0.52$ & $3.16 \pm 0.38$ \\
$\mathrm{Mg}(\mathrm{mg} / 100 \mathrm{~g})$ & $2.96 \pm 0.75$ & $3.04 \pm 0.35$ & $3.08 \pm 0.53$ & $2.14 \pm 0.39$ \\
Ca (mg/100g) & $1.85 \pm 0.30$ & $1.97 \pm 0.40$ & $2.07 \pm 0.82$ & $3.09 \pm 0.35$ \\
Total organic C $(\%)$ & $0.07 \pm 0.22$ & $2.86 \pm 0.29$ & $2.97 \pm 0.44$ & $0.60 \pm 0.09$ \\
Total organic matter $(\%)$ & $0.96 \pm 0.18$ & $0.82 \pm 0.26$ & $0.72 \pm 0.24$ & $73.02 \pm 0.70$ \\
Sand $(\%)$ & $77.92 \pm 0.88$ & $75.86 \pm 0.77$ & $74.07 \pm 0.75$ & $14.32 \pm 0.30$ \\
Silt $(\%)$ & $16.07 \pm 0.65$ & $13.12 \pm 0.38$ & $14.20 \pm 0.38$ & $12.20 \pm 0.68$ \\
Clay $(\%)$ & $5.43 \pm 0.26$ & $10.16 \pm 0.45$ & $11.02 \pm 0.54$ & $42.03 \pm 0.40$ \\
\hline Moisture content $(\%)$ & $60.07 \pm 0.85$ & $57.26 \pm 0.86$ & $46.12 \pm 0.55$ & \\
\hline
\end{tabular}

HARVEST (TMS 0505)

Mean \pm Standard Error 
TABLE 3: Nutrient contents in manihot esculenta (tms 0505) After harvest (control + pollution treatment)

\begin{tabular}{|lllll|}
\hline \multicolumn{5}{c|}{ CONCENTRATION $(\%)$} \\
PARAMETERS & 0 & 2 & 4 & 6 \\
$\mathrm{CHO}(\%)$ & $56.94 \pm 0.54$ & $44.07 \pm 0.42$ & $38.42 \pm 0.21$ & $31.67 \pm 0.20$ \\
Protein $(\%)$ & $49.76 \pm 0.44$ & $35.13 \pm 0.23$ & $29.86 \pm 0.19$ & $23.28 \pm 0.21$ \\
T.N $(\%)$ & $6.45 \pm 0.12$ & $5.30 \pm 0.21$ & $4.26 \pm 0.13$ & $4.02 \pm 0.14$ \\
T.Chlorophyll $(\mathrm{mg} / \mathrm{l})$ & $630.17 \pm 0.92$ & $482.51 \pm 0.81$ & $416.04 \pm 0.72$ & $295.34 \pm 0.54$ \\
$\mathrm{Ca}(\mathrm{mg} / 100 \mathrm{~g})$ & $14.21 \pm 0.21$ & $10.21 \pm 0.14$ & $9.06 \pm 0.10$ & $8.12 \pm 0.24$ \\
$\mathrm{Mg}(\mathrm{mg} / 10 \mathrm{~g})$ & $12.07 \pm 0.12$ & $9.23 \pm 0.40$ & $7.42 \pm 0.23$ & $7.02 \pm 0.21$ \\
$\mathrm{~K}(\mathrm{mg} / 100 \mathrm{~g})$ & $6.41 \pm 0.15$ & $6.72 \pm 0.18$ & $7.21 \pm 0.19$ & $7.30 \pm 0.12$ \\
$\mathrm{Na}(\mathrm{mg} / 100 \mathrm{~g})$ & $24.12 \pm 0.21$ & $22.21 \pm 0.30$ & $20.36 \pm 0.28$ & $19.85 \pm 0.19$ \\
$\mathrm{P}(\mathrm{mg} / 100 \mathrm{~g})$ & $15.72 \pm 0.12$ & $11.06 \pm 0.14$ & $9.24 \pm 0.11$ & $9.03 \pm 0.13$ \\
$\mathrm{Mn}(\mathrm{mg} / 100 \mathrm{~g})$ & $3.59 \pm 0.23$ & $4.07 \pm 0.31$ & $4.56 \pm 0.35$ & $4.86 \pm 0.40$ \\
$\mathrm{Zn}(\mathrm{mg} / 100 \mathrm{~g})$ & $0.36 \pm 0.02$ & $3.17 \pm 0.03$ & $3.66 \pm 0.02$ & $3.95 \pm 0.03$ \\
$\mathrm{~Pb}(\mathrm{mg} / 100 \mathrm{~g})$ & $0.47 \pm 0.04$ & $1.32 \pm 0.02$ & $1.56 \pm 0.03$ & $1.87 \pm 0.05$ \\
$\mathrm{Fe}(\mathrm{mg} / 100 \mathrm{~g})$ & $1.52 \pm 0.21$ & $2.36 \pm 0.35$ & $2.72 \pm 0.50$ & $2.80 \pm 0.23$ \\
$\mathrm{Cu}(\mathrm{mg} / 100 \mathrm{~g})$ & $0.93 \pm 0.08$ & $0.99 \pm 0.05$ & $1.32 \pm 0.12$ & $2.77 \pm 0.18$ \\
\hline
\end{tabular}

Mean \pm Standard Error

TABLE 4:Amelioration Treatment Variety Tms 0505 Soil Physico Chemical Properties After Harvest

\begin{tabular}{|c|c|c|c|c|c|c|c|c|c|c|c|c|}
\hline \multicolumn{4}{|c|}{ COMPOST } & \multicolumn{4}{|c|}{ NPK } & \multicolumn{4}{|c|}{ NPK+ COMPOST } & \multirow[b]{2}{*}{$6 \%$} \\
\hline $\begin{array}{l}\text { Parameter } \\
\mathrm{s}\end{array}$ & $0 \%$ & $2 \%$ & $4 \%$ & $6 \%$ & $0 \%$ & $2 \%$ & $4 \%$ & $6 \%$ & $0 \%$ & $2 \%$ & $4 \%$ & \\
\hline $\mathrm{pH}$ & $5.6 \pm$ & $5.6 \pm$ & $5.5 \pm$ & $5.5 \pm$ & $5.7 \pm$ & $5.5 \pm$ & $5.6 \pm$ & $5.5 \pm$ & $5.7 \pm$ & $5.5 \pm$ & $5.6 \pm$ & $5.6 \pm$ \\
\hline & 0.24 & 0.21 & 0.26 & 0.27 & 0.29 & 0.30 & 0.32 & 0.30 & 0.22 & 0.24 & 0.27 & 040 \\
\hline T.N (\%) & $0.27 \pm$ & $0.39 \pm$ & $0.36 \pm$ & $0.31 \pm$ & $0.26 \pm$ & $0.32 \pm$ & $0.27 \pm$ & $0.26 \pm$ & $0.28 \pm$ & $0.42 \pm$ & $0.38 \pm$ & $0.32 \pm$ \\
\hline & 0.01 & 0.02 & 0.03 & 0.02 & 0.03 & 0.04 & 0.03 & 0.04 & 0.02 & 0.02 & 0.04 & 0.03 \\
\hline AV.P & $6.31 \pm$ & $6.02 \pm$ & $5.86 \pm$ & $5.21 \pm$ & $6.33 \pm$ & $5.92 \pm$ & $5.30 \pm$ & $4.36 \pm$ & $6.32 \pm$ & $6.02 \pm$ & $5.86 \pm$ & $5.72 \pm$ \\
\hline$(m g / 100 g$ & 0.31 & 0.25 & 0.21 & 0.24 & 0.37 & 0.25 & 0.27 & 0.24 & 0.32 & 0.12 & 0.22 & 0.34 \\
\hline $\mathrm{Mn}$ & $1.06 \pm$ & $1.12 \pm$ & $1.18 \pm$ & $1.24 \pm$ & $1.07 \pm$ & $1.09 \pm$ & $1.13 \pm$ & $1.17 \pm$ & $1.06 \pm$ & $1.19 \pm$ & $1.27 \pm$ & $1.34 \pm$ \\
\hline$(\mathrm{mg} / 100 \mathrm{~g}$ & 0.12 & 0.10 & 0.11 & 0.16 & 0.20 & 0.22 & 0.18 & 0.16 & 0.12 & 0.23 & 0.24 & 0.25 \\
\hline $\begin{array}{l}\mathrm{Zn} \\
(\mathrm{mg} / 100 \mathrm{~g}\end{array}$ & $\begin{array}{l}0.84 \pm \\
0.01\end{array}$ & $\begin{array}{l}0.96 \pm \\
0.02\end{array}$ & $\begin{array}{l}0.41 \pm \\
0.03\end{array}$ & $\begin{array}{l}0.34 \pm \\
0.02\end{array}$ & $\begin{array}{l}0.87 \pm \\
0.05\end{array}$ & $\begin{array}{l}0.72 \pm \\
0.06\end{array}$ & $\begin{array}{l}0.36 \pm \\
0.01\end{array}$ & $\begin{array}{l}0.28 \pm \\
0.02\end{array}$ & $\begin{array}{l}0.86 \pm \\
0.03\end{array}$ & $\begin{array}{l}0.95 \pm \\
0.07\end{array}$ & $\begin{array}{l}0.83 \pm \\
0.09\end{array}$ & $\begin{array}{l}0.64 \pm \\
0.05\end{array}$ \\
\hline $\begin{array}{l}\mathrm{Cu} \\
(\mathrm{mg} / 100 \mathrm{~g}\end{array}$ & $\begin{array}{l}1.57 \pm \\
0.22\end{array}$ & $\begin{array}{l}1.84 \pm \\
0.12\end{array}$ & $\begin{array}{l}1.72 \pm \\
0.13\end{array}$ & $\begin{array}{l}1.63 \pm \\
0.24\end{array}$ & $\begin{array}{l}1.54 \pm \\
0.30\end{array}$ & $\begin{array}{l}1.65 \pm \\
0.25\end{array}$ & $\begin{array}{l}1.54 \pm \\
0.18\end{array}$ & $\begin{array}{l}1.36 \pm \\
0.12\end{array}$ & $\begin{array}{l}1.57 \pm \\
0.17\end{array}$ & $\begin{array}{l}1.94 \pm \\
0.30\end{array}$ & $\begin{array}{l}1.85 \pm \\
0.25\end{array}$ & $\begin{array}{l}1.77 \pm \\
0.32\end{array}$ \\
\hline $\mathrm{Pb}(\mathrm{mg} / 10$ & $0.89 \pm$ & $1.74 \pm$ & $1.67 \pm$ & $1.52 \pm$ & $0.88 \pm$ & $1.52 \pm$ & $1.46 \pm$ & $1.35 \pm$ & $0.87 \pm$ & $1.82 \pm$ & $1.76 \pm$ & $1.64 \pm$ \\
\hline $0 \mathrm{~g})$ & 0.05 & 0.15 & 0.18 & 0.23 & 0.16 & 0.25 & 0.28 & 0.30 & 0.08 & 0.19 & 0.16 & 0.42 \\
\hline $\mathrm{Fe}$ & $1.42 \pm$ & $2.40 \pm$ & $2.66 \pm$ & $2.79 \pm$ & $1.42 \pm$ & $2.36 \pm$ & $2.47 \pm$ & $2.58 \pm$ & $1.44 \pm$ & $2.61 \pm$ & $2.77 \pm$ & $2.95 \pm$ \\
\hline$(\mathrm{mg} / 100 \mathrm{~g}$ & 0.15 & 0.23 & 0.30 & 0.14 & 0.23 & 0.36 & 0.25 & 0.28 & 0.25 & 0.28 & 0.30 & 0.21 \\
\hline $\begin{array}{l}\mathrm{Cd} \\
(\mathrm{mg} / 100 \mathrm{~g}\end{array}$ & $\begin{array}{l}0.76 \pm \\
0.26\end{array}$ & $\begin{array}{l}0.84 \pm \\
0.32\end{array}$ & $\begin{array}{l}0.90 \pm \\
0.40\end{array}$ & $\begin{array}{l}1.20 \pm \\
0.14\end{array}$ & $\begin{array}{l}0.75 \pm \\
0.22\end{array}$ & $\begin{array}{l}0.77 \pm \\
0.30\end{array}$ & $\begin{array}{l}0.82 \pm \\
0.24\end{array}$ & $\begin{array}{l}0.94 \pm \\
0.25\end{array}$ & $\begin{array}{l}0.76 \pm \\
0.32\end{array}$ & $\begin{array}{l}0.94 \pm \\
0.84\end{array}$ & $\begin{array}{l}1.12 \pm \\
0.15\end{array}$ & $\begin{array}{l}1.30 \pm \\
0.18\end{array}$ \\
\hline $\begin{array}{l}\mathrm{Cr} \\
(\mathrm{mg} / 100 \mathrm{~g}\end{array}$ & $\begin{array}{l}0.88 \pm \\
0.02\end{array}$ & $\begin{array}{l}0.92 \pm \\
0.03\end{array}$ & $\begin{array}{l}1.07 \pm \\
0.42\end{array}$ & $\begin{array}{l}1.12 \pm \\
0.24\end{array}$ & $\begin{array}{l}0.89 \pm \\
0.04\end{array}$ & $\begin{array}{l}0.93 \pm \\
0.05\end{array}$ & $\begin{array}{l}1.02 \pm \\
0.30\end{array}$ & $\begin{array}{l}1.07 \pm \\
0.24\end{array}$ & $\begin{array}{l}0.87 \pm \\
0.08\end{array}$ & $\begin{array}{l}0.96 \pm \\
0.95\end{array}$ & $\begin{array}{l}1.18 \pm \\
0.22\end{array}$ & $\begin{array}{l}1.27 \pm \\
0.18\end{array}$ \\
\hline $\begin{array}{l}\mathrm{K} \\
(\mathrm{mg} / 100 \mathrm{~g}\end{array}$ & $\begin{array}{l}6.42 \pm \\
0.40\end{array}$ & $\begin{array}{l}5.76 \pm \\
0.30\end{array}$ & $\begin{array}{l}5.24 \pm \\
0.26\end{array}$ & $\begin{array}{l}5.06 \pm \\
0.30\end{array}$ & $\begin{array}{l}6.44 \pm \\
0.40\end{array}$ & $\begin{array}{l}5.07 \pm \\
0.28\end{array}$ & $\begin{array}{l}4.86 \pm \\
0.38\end{array}$ & $\begin{array}{l}4.70 \pm \\
0.28\end{array}$ & $\begin{array}{l}6.43 \pm \\
0.42\end{array}$ & $\begin{array}{l}6.09 \pm \\
0.50\end{array}$ & $\begin{array}{l}5.87 \pm \\
0.48\end{array}$ & $\begin{array}{l}5.63 \pm \\
0.38\end{array}$ \\
\hline $\begin{array}{l}\mathrm{Na} \\
(\mathrm{mg} / 100 \mathrm{~g}\end{array}$ & $\begin{array}{l}27.6 \pm \\
0.12\end{array}$ & $\begin{array}{l}30.4 \pm \\
0.60\end{array}$ & $\begin{array}{l}34.0 \pm \\
0.50\end{array}$ & $\begin{array}{l}36.0 \pm \\
0.30\end{array}$ & $\begin{array}{l}27.4 \pm \\
0.28\end{array}$ & $\begin{array}{l}29.0 \pm \\
0.21\end{array}$ & $\begin{array}{l}32.1 \pm \\
0.26\end{array}$ & $\begin{array}{l}33.9 \pm \\
0.30\end{array}$ & $\begin{array}{l}28.0 \pm \\
0.26\end{array}$ & $\begin{array}{l}33.0 \pm \\
0.30\end{array}$ & $\begin{array}{l}36.1 \pm \\
0.25\end{array}$ & $\begin{array}{l}39.2 \pm \\
0.12\end{array}$ \\
\hline $\begin{array}{l}\mathrm{Mg} \\
(\mathrm{mg} / 100 \mathrm{~g}\end{array}$ & $\begin{array}{l}2.95 \pm \\
0.12\end{array}$ & $\begin{array}{l}3.62 \pm \\
0.22\end{array}$ & $\begin{array}{l}3.70 \pm \\
0.25\end{array}$ & $\begin{array}{l}3.96 \pm \\
0.30\end{array}$ & $\begin{array}{l}2.94 \pm \\
0.15\end{array}$ & $\begin{array}{l}3.01 \pm \\
0.26\end{array}$ & $\begin{array}{l}3.31 \pm \\
0.28\end{array}$ & $\begin{array}{l}3.47 \pm \\
0.30\end{array}$ & $\begin{array}{l}2.95 \pm \\
0.25\end{array}$ & $\begin{array}{l}3.77 \pm \\
0.24\end{array}$ & $\begin{array}{l}3.98 \pm \\
0.32\end{array}$ & $\begin{array}{l}4.10 \pm \\
0.50\end{array}$ \\
\hline $\begin{array}{l}\mathrm{Ca} \\
(\mathrm{mg} / 100 \mathrm{~g}\end{array}$ & $\begin{array}{l}2.02 \pm \\
0.12\end{array}$ & $\begin{array}{l}2.41 \pm \\
0.24\end{array}$ & $\begin{array}{l}3.60 \pm \\
0.28\end{array}$ & $\begin{array}{l}3.86 \pm \\
0.28\end{array}$ & $\begin{array}{l}2.03 \pm \\
0.32\end{array}$ & $\begin{array}{l}2.36 \pm \\
0.11\end{array}$ & $\begin{array}{l}2.94 \pm \\
0.15\end{array}$ & $\begin{array}{l}3.12 \pm \\
0.25\end{array}$ & $\begin{array}{l}2.04 \pm \\
0.28\end{array}$ & $\begin{array}{l}3.02 \pm \\
0.30\end{array}$ & $\begin{array}{l}3.71 \pm \\
0.25\end{array}$ & $\begin{array}{l}3.96 \pm \\
0.28\end{array}$ \\
\hline TOC (\%) & $\begin{array}{l}0.12 \pm \\
0.01\end{array}$ & $\begin{array}{l}2.17 \pm \\
0.23\end{array}$ & $\begin{array}{l}2.96 \pm \\
0.30\end{array}$ & $\begin{array}{l}3.84 \pm \\
0.28\end{array}$ & $\begin{array}{l}0.11 \pm \\
0.08\end{array}$ & $\begin{array}{l}2.10 \pm \\
0.24\end{array}$ & $\begin{array}{l}2.42 \pm \\
0.27\end{array}$ & $\begin{array}{l}2.61 \pm \\
0.28\end{array}$ & $\begin{array}{l}0.12 \pm \\
0.18\end{array}$ & $\begin{array}{l}2.24 \pm \\
0.12\end{array}$ & $\begin{array}{l}3.07 \pm \\
0.24\end{array}$ & $\begin{array}{l}3.92 \pm \\
0.52\end{array}$ \\
\hline TOM (\%) & $0.52 \pm$ & $1.14 \pm$ & $0.62 \pm$ & $0.43 \pm$ & $0.54 \pm$ & $1.02 \pm$ & $0.36 \pm$ & $0.20 \pm$ & $0.54 \pm$ & $1.19 \pm$ & $0.83 \pm$ & $0.70 \pm$ \\
\hline Sand $(\%)$ & $\begin{array}{l}77.0 \pm \\
0.80\end{array}$ & $\begin{array}{l}75.1 \pm \\
0.88\end{array}$ & $\begin{array}{l}74.0 \pm \\
0.90\end{array}$ & $\begin{array}{l}73.1 \pm \\
0.56\end{array}$ & $\begin{array}{l}77.8 \pm \\
0.45\end{array}$ & $\begin{array}{l}75.8 \pm \\
0.57\end{array}$ & $\begin{array}{l}95.1 \pm \\
0.90\end{array}$ & $\begin{array}{l}75.0 \pm \\
0.50\end{array}$ & $\begin{array}{l}77.5 \pm \\
0.62\end{array}$ & $\begin{array}{l}75.2 \pm \\
0.70\end{array}$ & $\begin{array}{l}74.1 \pm \\
0.60\end{array}$ & $\begin{array}{l}74.0 \pm \\
0.80\end{array}$ \\
\hline Silt (\%) & $\begin{array}{l}16.5 \pm \\
0.32\end{array}$ & $\begin{array}{l}12.0 \pm \\
0.24\end{array}$ & $\begin{array}{l}14.0 \pm \\
0.26\end{array}$ & $\begin{array}{l}14.0 \pm \\
0.25\end{array}$ & $\begin{array}{l}17.2 \pm \\
0.30\end{array}$ & $\begin{array}{l}12.2 \pm \\
0.25\end{array}$ & $\begin{array}{l}12.3 \pm \\
0.30\end{array}$ & $\begin{array}{l}13.4 \pm \\
0.24\end{array}$ & $\begin{array}{l}16.7 \pm \\
0.25\end{array}$ & $\begin{array}{l}12.6 \pm \\
0.15\end{array}$ & $\begin{array}{l}13.6 \pm \\
0.15\end{array}$ & $\begin{array}{l}13.9 \pm \\
0.16\end{array}$ \\
\hline Clay $(\%)$ & $\begin{array}{l}5.50 \pm \\
0.24\end{array}$ & $\begin{array}{l}10.5 \pm \\
0.50\end{array}$ & $\begin{array}{l}10.6 \pm \\
0.60\end{array}$ & $\begin{array}{l}11.1 \pm \\
0.45\end{array}$ & $\begin{array}{l}5.41 \pm \\
0.24\end{array}$ & $\begin{array}{l}10.06 \pm 0 \\
.60\end{array}$ & $\begin{array}{l}11.02 \pm 0 \\
.50\end{array}$ & $\begin{array}{l}11.09 \pm 0 \\
.65\end{array}$ & $\begin{array}{l}5.24 \pm 0 \\
30\end{array}$ & $\begin{array}{l}10.46 \pm 0 \\
.60\end{array}$ & $\begin{array}{l}10.52 \pm 0 \\
.50\end{array}$ & $\begin{array}{l}11.80 \pm 0 \\
.60\end{array}$ \\
\hline $\begin{array}{l}\text { Moisture } \\
(\%)\end{array}$ & $\begin{array}{l}61.21 \pm 0 \\
.70\end{array}$ & $\begin{array}{l}62.07 \pm 0 \\
.80\end{array}$ & $\begin{array}{l}56.07 \pm 0 \\
.65\end{array}$ & $\begin{array}{l}56.01 \pm 0 \\
.42\end{array}$ & $\begin{array}{l}61.30 \pm 0 \\
.62\end{array}$ & $\begin{array}{l}60.02 \pm 0 \\
.25\end{array}$ & $\begin{array}{l}53.42 \pm 0 \\
.45\end{array}$ & $\begin{array}{l}50.03 \pm 0 \\
.10\end{array}$ & $\begin{array}{l}5.24 \pm \\
0.46\end{array}$ & $\begin{array}{l}10.46 \pm 0 \\
.50\end{array}$ & $\begin{array}{l}10.52 \pm 0 \\
.82\end{array}$ & $\begin{array}{l}11.80 \pm 0 \\
.38\end{array}$ \\
\hline
\end{tabular}

Mean \pm standard error 

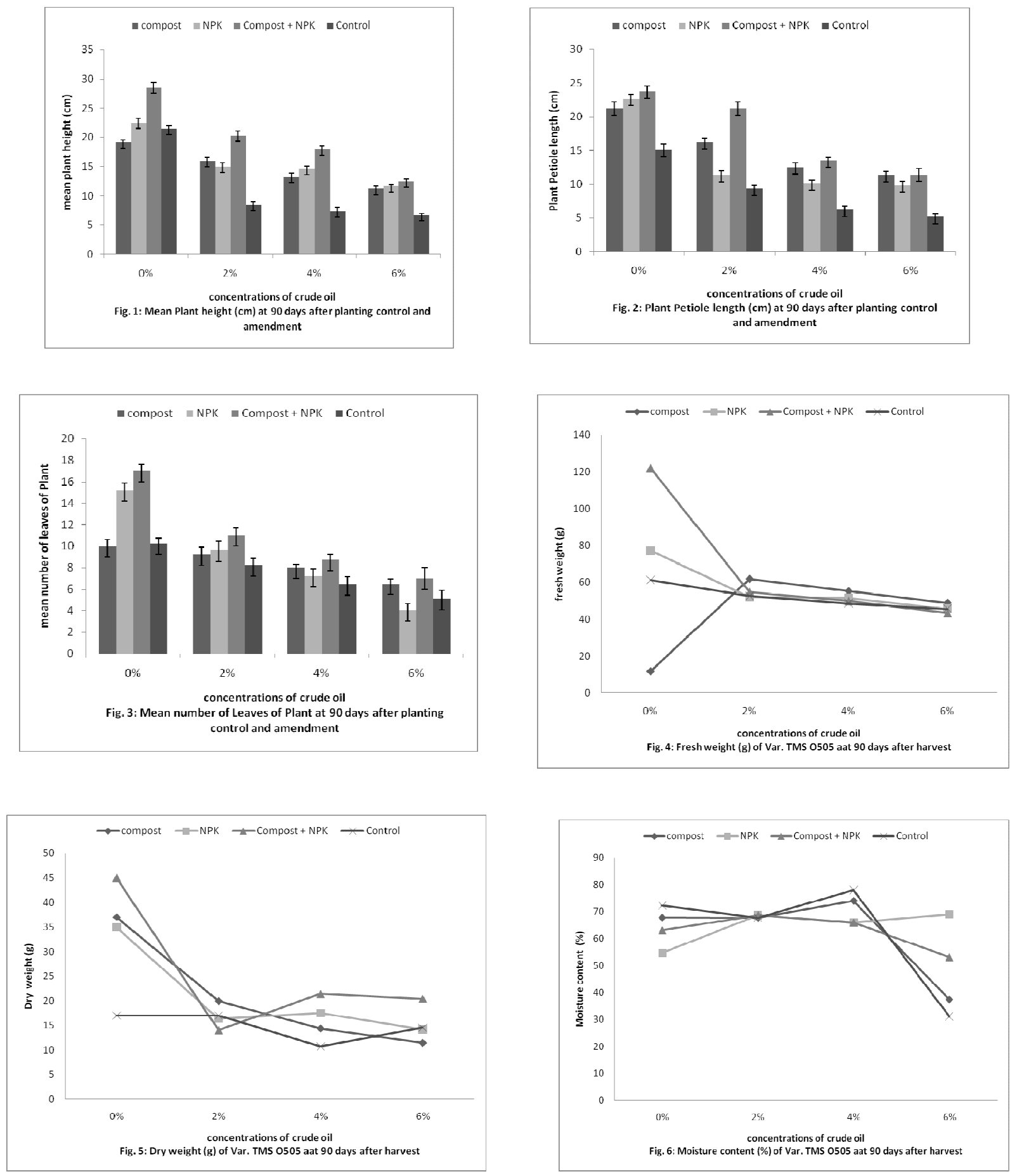

Conclusion: Both organic and inorganic fertilizers have proved to be very beneficial in ameliorating crude oil polluted soil by adding nutrients to the soil which creates an enabling environment for the microorganisms to thrive, thus degrading the toxic hydrocarbons in soil, making it harmless and creating an environment that supports plant growth and development. This study shows that the use of

Centrosema pubescens and inorganic fertilizer as single or combined treatment would improve the soil condition and enhance the growth of Manihot esculenta in crude oil polluted soil.

\section{REFERENCES}

Al-Azab, M., EI-Shorbagy, W. And Al-Ehias, S.,(2005). Oil pollution and its environmental. 
Impact the Arabian Gulf Region; Measurement of photosynthesis and respiration in oil polluted soil. Books.google.com.ng/books ISBN=0444520600

Alexander, S.K., Webb, J.W. (1987). Relationship of Spartina alterniflora growth to sediment oil content following an oil spill. Proceedings of 1987. International oil spill conference. America petroleum Institute. Washington DC. Pp 445-449

Allison, C. E., (1965). Methods of soil analysis Agronomy, 9 (2): 1367-1378.

Anyanwu, D.I. and Tanee, F.B.G.(2008). Tolerance of cassava (var. TMS 30572) to different concentrations of post-planting crude oil pollution. Nigerian journal of botany.21(1):203-207.

A.O.A.C., (1975). Association of official analytical chemist. Methods of Analysis (10th, 11th and 12th Edition), Washington DC., U.S.A.

Audrey,G., (2001). A report on Nigeria; Petroleum pollution and poverty in the Niger Delta.

Amnesty international, Canada. Index number: AFR 44/017

Carls, M.G. (2001). Persistence of oiling in mussel beds after the Exxon Valdez oil spill.Marine Environmental research.51:167-190

Davis, J.G., Wilson, C.R. (2005). Choosing a soil amendment. Colorado University, Cooperative Extension Horticulture 7:235

Fernet, J. L. (2008). Plant bacterial inoculants to remediate hydrocarbon polluted soil. (M.Sc Thesis, University of Saskatchewan, Saskatoon).

Georgewill, O.A. and Nwankwaola, R.N.P. (2006). The effects of prolonged exposure of Nigerian crude oil reproductive hormones of rats. Journal of Applied Zoology and Environmental Biology. 8:16-19

Inoni, O. E., (2006). The effect of oil spillage on crop yield and farm income in Delta. www.agr.hr/jcea/issues/jcea71/pdf/jcea71-6 .pdf.

Lee, K., Doe, K.G., Lee, L.E.J., Suidan, M.T. \& Venosa, A.D. (2001). Remediation of an oil contaminated experimental freshwater wetland. Habitat recovery and toxicity
reduction.Proceedings of the 2001 International Oil Spill Conference. American Petroleum Institute, Washington DC

NLNG (Nigeria Liquified Natural Gas) (1995). Environmental Impact Assessment Report. Vol. 10

Odokuma, L.O., and Ibor, M.N. (2002). Nitrogen fixing bacteria enhanced bioremediation of a crude oil polluted soil. Global Journal of Pure and Applied Science. 8(4): 455- 468.

Offor, U.S., and Akonye, L. A. (2006). Amendment of crude oil contaminated soil with sawdust and Chromolena leaves for optimum plant protection. African Journal of Biotech. 5 (9):770-774.

Ogbogbodo, I.A.,Azenabor, U.F. and Osemwota, I.O. (2005). Amelioration of crude oil Polluted soil with Poultry Manure and effect on growth of Maize and some soil properties. Journal of Plant Nutrition 28(1):21-32

Pezeshki, S.R., Hester, M.W., Lin,Q. And Nyman, J.A.(2000). The effects of oil spill and clean-up on dominant US Gulf coast march microphytes; a review. Environmental pollution 108: 129139.

Renalt, S., Zwiazek, J. J., Fung, M. and Tuttle, S., (2000). Germination, growth and gas exchange of selected boreal forest seedlings in soil containing oil sand tailings. Environmental pollution, 107(3):357-365.

Stewart, E.A., Grimshaw, H.M., Parkinson, J.A, Quarmby, C. (1974). Chemical Analysis of Ecological Materials. Black well Publications, London.

Tanee, F.B.G. and Akonye, L.A.(2009). Effectiveness of Vigna unguiculata as a phytoremediation plant in the remediation of crude oil polluted soil for cassava (Manihot esculenta, Crantz) cultivation. J. Apl. Sci. Environ. Manage., 13(1): 43-47.

Tanee, F.B.G. \& Anyanwu, D.I. (2007). Comparative studies of the growth and yield of two cassava lines (TMS 30572 and TMS 30555) in a crude oil polluted habitat. Scientia Africana. An International Journal of Pure and Applied Sciences. 6(1): 81-85.

Udo, E.J., and Ogunwale, J. A., (1986). Laboratory Manual for the Analysis Soil, Plant and Water Samples. $2^{\text {nd }}$ Edition, University Press, Ibadan, Nigeria. 
Udo, E.J., and Oputa, C. O., (1984). Some studies on the effect of crude oil pollution of soil on plant growth. Journal of Biol. and A pplied Chemistry, 26(29):3-14.
Vwioko, D.C. and Fashemi, D. S.,(2005). Growth response of Ricinus communis $\mathrm{L}$ (Castor oil) in spent lubricating oil polluted soil.. Journal Applied Bcl. Environ Mgt.9(2):73-79 